\title{
On the manifestation of stimulus-directed behavior in the rat
}

\author{
CHARLES R. CROWELL, THOMAS P. BERNHARDT, and PATRICK MOSKAL \\ University of Notre Dame, Notre Dame, Indiana 46556
}

\begin{abstract}
The results of a three-phase autoshaping experiment with rats is reported. In Phase 1 , subjects were exposed either to a paired or an unpaired arrangement of a visual CS and food. These treatments produced no differential contact with the visual stimulus, but paired rats were found to display greater approach to the site of food delivery than were unpaired subjects during the CS. In Phase 2, all subjects were given identical discrete-trials (FR 6) training with a different visual stimulus as an $\mathrm{S}^{\mathrm{D}}$. In the final test stage, a choice procedure was employed, involving the simultaneous presentation of both CS and $\mathrm{S}^{\mathrm{D}}$. Percent responding to the CS was greater for paired than for unpaired rats in this phase. These findings were interpreted as support for the view that the form of terminal contact with a signal for food is dependent upon the nature of the learned or innate behaviors that compose the organism's normal foodgetting sequence.
\end{abstract}

For several years, a program of research in our laboratory has focused upon investigations with rats of the consequences for directed action of exposure to various environmental stimulus-reinforcer relations. This research has taken two paths. In one, stimulus-reinforcer correlations were embedded within instrumental discrimination paradigms through the use of feature-positive (FP) or feature-negative (FN) learning arrangements. In these situations, the multielement discriminanda shared common features but were differentiated by a discrete stimulus localized in either S+ (FP) or S- (FN). Our findings with these paradigms using rats have been largely consistent with those of previous investigations utilizing other species (see Hearst, 1978, for a review). We have found within-stimulus changes in the distribution of responding to the various elements of S+ and $\mathrm{S}-$ during the course of discrimination training (Crowell \& Bernhardt, 1979). Over sessions, FP subjects have exhibited an increasing tendency to direct their responding away from the common elements toward the differentiating feature in $\mathbf{S}+$, whereas a progressive decline in proportionate responding to the distinguishing element in $\mathrm{S}-$ has been noted with the FN condition. Since the reinforcement contingencies in effect during $\mathrm{S}+$ and S- stimuli in these tasks did not differentially affect responding to any elements, such redirected responding. commonly has been attributed to the response-directing effects of the differentiating element's positive (FP) or negative (FN) relation to food delivery (Hearst, 1975, 1978; Hearst \& Jenkins, 1974).

The other path of our research has involved the

The authors wish to express their gratitude to Ralph Gilpatrick and J. V. Lupo for assistance in various phases of this research. Requests for reprints should be sent to Charles R. Crowell, Department of Psychology, University of Notre Dame, Notre Dame, Indiana 46556. analysis of stimulus-reinforcer effects within a context that has come to be known as the autoshaping paradigm. The prototypical example of such an arrangement was reported by Brown and Jenkins (1968). In this study, pigeons developed a tendency to approach and contact a lighted response key even though reinforcement was not dependent upon the organism's behavior and was contingent only upon prior stimulus presentation. Subsequent research has clearly revealed autoshaping to be a robust phenomenon that is critically dependent upon positive stimulus-reinforcer correlations (see Schwartz \& Gamzu, 1977, for a review).

Our own efforts in this regard with rats have been less successful than would appear warranted on the basis of available evidence. A number of recent investigations with this species have documented the development of behaviors that involved coming into contact with a CS (invariably a protruding lever) that was consistently followed by reinforcer delivery (e.g., Boakes, 1977; Locurto, Terrace, \& Gibbon, 1976; Peterson, Ackil, Frommer, \& Hearst, 1972). However, using a lighted response key mounted flush with a side wall as the stimulus, we have failed on several occasions to show differential levels of contact with the CS in two groups exposed to either paired or unpaired arrangements of the stimulus and food. The purpose of the present report is to exemplify our difficulties in this connection and to demonstrate the consequences of a factor that can lead to differential signal-directed responding under these circumstances.

\section{METHOD}

\section{Subjects}

The subjects were 12 naive Sprague-Dawley albino males weighing approximately $320-350 \mathrm{~g}$ at the start of the study. They were housed individually under conditions of a 12-h 
light/dark cycle, and all had free access to water in the colony throughout the experiment.

\section{Apparatus}

The experimental chamber for this study was a rectangular box with inside dimensions measuring $23 \mathrm{~cm}$ from side to side, $32 \mathrm{~cm}$ from front to back, and $27 \mathrm{~cm}$ from floor to ceiling. The front wall and top were composed of Plexiglas, the sides and back of aluminum, and the floor of hardware cloth. Three rectangular openings, $3.5 \times 3.8 \mathrm{~cm}$, were cut in one side wall $3 \mathrm{~cm}$ apart and $2 \mathrm{~cm}$ above the floor, the innermost being located $4 \mathrm{~cm}$ from the back wall. Response keys just large enough to cover each rectangular opening were mounted on the outside surface of the box. Each key consisted of a strip of thin translucent milk-white plastic, hinged at the top and positioned so that virtually any contact with the key by a rat would activate a sensitive microswitch. Each key could be illuminated from behind with one of two intensities of white light produced by separate Lehigh Valley Tri-Color lamps (Model 114-01) mounted directly behind each key. The more intense level was obtained by connecting all three bulbs within each lamp (color caps removed) in parallel to a $26-\mathrm{V}$ power supply. A less intense light was produced by switching a $150-0 h m$ resistor in series with the three-bulb parallel network. A food tray $8 \mathrm{~cm}$ high, $4.5 \mathrm{~cm}$ wide, and $4 \mathrm{~cm}$ deep was mounted on the side wall opposite the response keys, directly across from the center key. Access to the tray was provided by a $3.5 \times 3.8 \mathrm{~cm}$ opening in the wall $2 \mathrm{~cm}$ above the floor. The food tray was continuously illuminated from above by a standard $28-\mathrm{V}$ bulb positioned behind a milkwhite diffusing screen mounted in the upper portion of the tray. Noyes precision food pellets $(.045 \mathrm{~g})$ were delivered to the tray from behind the diffusing screen by a Gerbrands pellet dispenser (Model 65100). Programming and recording of events was accomplished by a Digital Equipment Corporation PDP-8/I computer located in another room.

\section{Procedure}

The rats were acclimated to the laboratory for 7 days following their arrival; during this time they had free access to food and water. All rats subsequently were food deprived and reduced to $85 \%$ of their baseline weights, a level that was maintained throughout the study. The 1 st day of treatment for all rats consisted of training them to eat from the food cup by means of a procedure that involved 15-20 noncontingent food presentations randomly placed in a 30-min session.

On the following day, Phase 1 of the experiment was initiated. The purpose of this phase was to expose two groups to different stimulus-reinforcer relationships in the absence of any response contingency. This stage consisted of seven daily sessions of 30 conditioning trials each. For one group (paired), conditioning involved forward pairings of the center key light with food. Throughout this phase the brighter level of illumination served as the CS. Each pairing consisted of a $20-\mathrm{sec}$ presentation of the light, followed immediately by a single food pellet. The intertrial interval (ITI) was $2 \mathrm{~min} \pm 30 \mathrm{sec}$. A second group (unpaired) experienced explicitly unpaired presentations of the CS and food during this phase. The total number of CS and food occurrences was the same for this group as for the paired subjects, but stimulus presentations were arranged so that a minimum interval of $30 \mathrm{sec}$ separated either onset or offset of the CS from food. Otherwise, stimulus presentations were randomly determined, with the restriction that no more than two of the same kind could occur in a row. The interstimulus interval (ISI) (offset to onset) for this group was $1 \mathrm{~min} \pm 15 \mathrm{sec}$. During this phase, number of responses to the CS key, as well as number of food-cup entries, were recorded for both CS and ITI (or ISI) periods.

Phase 2, for all subjects, consisted of six daily sessions of discrete-trials instrumental response training. The purpose of this phase was to establish a dimly illuminated center key light as an $S D$ for pressing. Each session involved 60 trials spaced at
1 -min $( \pm 30 \mathrm{sec})$ intervals. On the 1 st day of this phase, rats were shaped to press, with nose or paw, the dimly illuminated center key while a continuous reinforcement procedure was in effect during each trial. For the next session, the response requirement for each trial was increased to FR 3 . The last 4 days of Phase 2 entailed FR 6 training.

The final stage of the study was a test phase intended to pit the directed response-evoking properties of the $S^{D}$ from Phase 2 against those of the CS from Phase 1. A variant of a concurrentchoice procedure was used for this purpose. According to this procedure, every subject was administered a single session consisting of 30 discrete extinction trials spaced in the same manner as in Phase 2. Each trial involved illumination of the center and one side key. The center key was always dimly lighted with the $S^{D}$ from Phase 2, whereas a side key contained the brighter CS light from Phase 1. Across the trials in this test session, the CS light appeared equally often on each side key (i.e., left and right) according to a random sequence. A total of six responses distributed to one or both illuminated keys terminated a trial without reinforcement in this session. Responses to the unlighted key were ignored. The maximum trial duration in this phase was $20 \mathrm{sec}$; after this time, trials were ended automatically without reward.

\section{RESULTS}

Figure 1 depicts the group mean total numbers of presses to the lighted CS key summed over the 30 trials in each session of Phase 1. It is apparent from this graph that the pressing behaviors of paired and unpaired groups were virtually identical throughout this conditioning stage. Moreover, it is clear that both groups exhibited a marked decrease in CS pressing over sessions. These observations were confirmed by the results of a groups by sessions ANOVA applied to the data upon which Figure 1 is based. Only the sessions effect emerged significant in this analysis $[F(6,60)=6.49, p<.01]$.

Figure 2 illustrates the mean food-cup entries (i.e., head pokes) per second for paired and unpaired groups

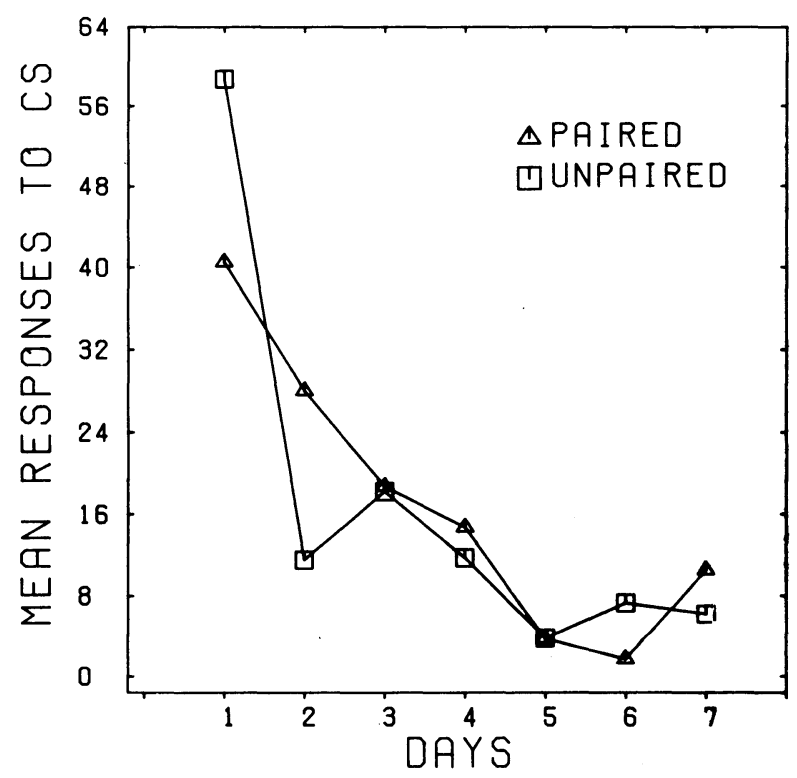

Figure 1. Mean total number of presses to the lighted CS key summed over the 30 trials in each session of Phase 1 for paired and unpaired groups. 
during CS an ITI periods as a function of sessions in Phase 1. This graph shows that the pattern of CS head poking over sessions was different for the two groups. Unpaired subjects exhibited their highest levels of CS food-cup entries initially and showed a general decrease in this behavior over sessions thereafter. In contrast, paired rats displayed a slightly increasing tendency to enter the food cup during the CS throughout this phase. Considering ITI periods, head poking generally was greater for paired than for unpaired rats, and both groups exhibited decreasing tendencies to enter the food cup over sessions in this period.

The pattern of effects in Figure 2 was evaluated by means of separate groups by sessions ANOVAs applied to the CS and ITI food-cup entry data. In both analyses, the groups effects were significant [both $\operatorname{Fs}_{s}(1,10) \geqslant 7.82$, ps $<.05]$. The sessions effect was significant only for the ITI measures $[F(6,60)=3.24, p<.01]$, and the Groups by Sessions interaction was significant only for the CS data $[F(6,60)=4.38, p<.01]$. The latter effect resulted from a significant decrease over sessions in head pokes during the $\mathrm{CS}$ for the unpaired group $[\mathrm{F}(6,30)=$ $4.62, \mathrm{p}<.01]$ and a marginally significant increase in this behavior during conditioning for paired subjects $[F(6,30)=2.40, p=.05]$. Moreover, paired and unpaired groups, while not significantly different in terms of head poking during the CS on Session 1, were different by the end of this phase $[t(10)=2.63, p<.05]$.

During Phase 2, all subjects acquired the FR 6 instrumental response without difficulty. Throughout this stage, there were no significant differences between paired and unpaired groups in the mean time taken to complete the FR requirements, averaged over trials within each session.

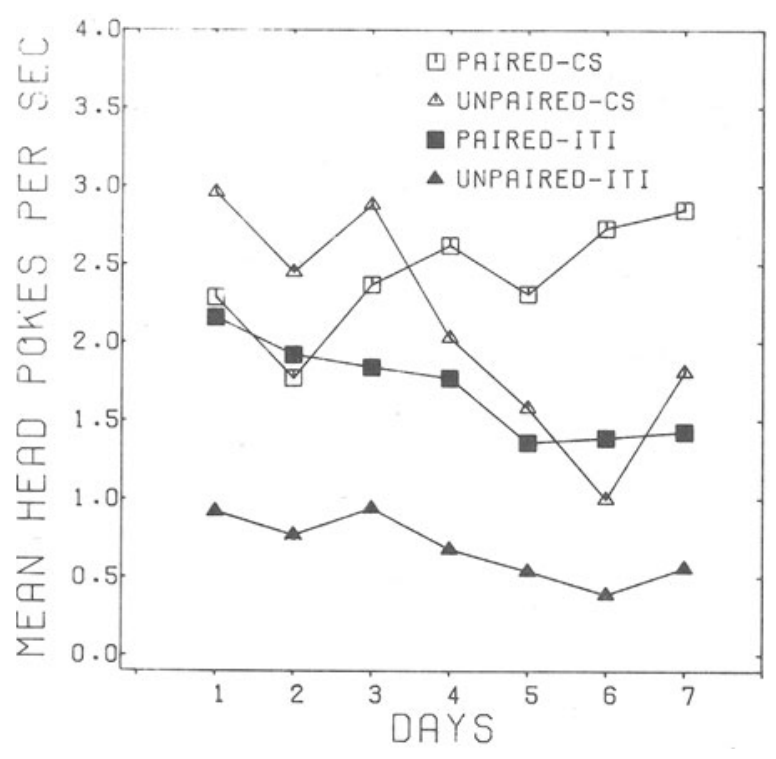

Figure 2. Mean head pokes per second during CS and ITI periods as a function of groups and sessions in Phase 1.

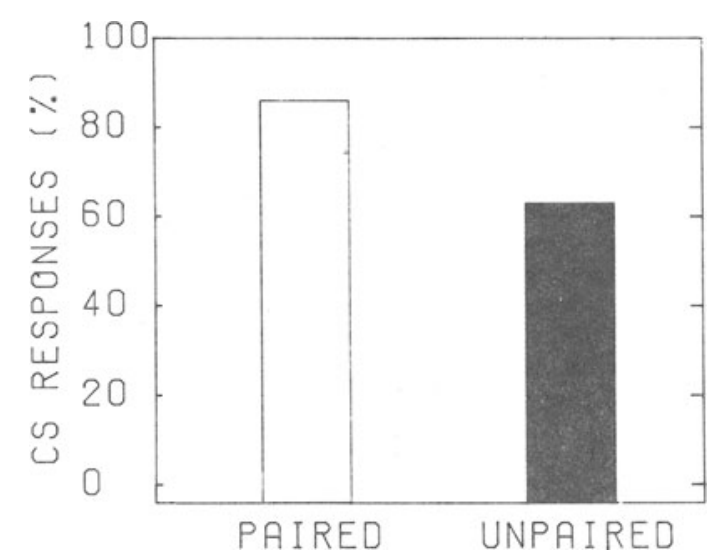

Figure 3. Mean percent responding to the CS key for paired and unpaired groups in Phase 3.

Figure 3 summarizes the outcome of the final test phase in terms of percent responding to the Phase 1 CS key for each group. It is clear from this figure that paired subjects directed nearly all of their responding toward the CS key, whereas unpaired rats distributed their pressing more evenly between the two alternative stimuli (CS and SD). The apparent difference between the groups on this measure was significant $[F(1,10)=$ $7.39, \mathrm{p}<.05]$.

\section{DISCUSSION}

Phase 1 of the present study conformed to procedures typical of most experiments on autoshaping. In contrast to the usual outcome of such studies, however, forward pairings of a visual stimulus and food did not lead to levels of contact with the CS that were significantly different from those engendered by an unpaired arrangement of stimuli. In fact, both groups exhibited equivalent decreases in CS contact over the course of Phase 1 conditioning.

The question raised by this finding, therefore, pertains to the reasons why the paired treatment in this and several other studies from our laboratory did not lead to the development of tendencies to contact the CS, such as those that have been obtained in numerous other experiments employing similar stimulus-reinforcer relations. One possible explanation of this outcome may be that the present paired and unpaired conditions were both functionally ineffective learning arrangements. However, the fact that these conditions were observed to have differential consequences for food-cup entry behavior in Phase 1 is not consistent with this interpretation. That is, headpoking during the CS, while initially the same for both groups, was significantly greater for paired than for unpaired subjects by the end of the first phase. This finding seems clearly to indicate that the associative consequences of paired and unpaired treatments were not the same in Phase 1, despite nondifferential contact with the CS in these groups. A similar result with respect to food-cup entry behavior has been reported by Boakes (1977), among others, and such an outcome may be taken as evidence that a CS, when paired with foed, can acquire control over behavior directed at the site of reinforcer delivery.

Alternatively, one could argue that the increased tendency to approach the food delivery site exhibited by paired rats in Phase 1 represented a pattern of behavior that was incompatible with approach to (and therefore contact with) the CS. Two 
lines of investigation provide a basis for arguing against the plausibility of this account of the present Phase 1 outcomes. The first, reported by Boakes (1977), reveals that under some circumstances both tendencies to approach the site of food delivery and to make contact with an illuminated lever serving as a signal for food can be exhibited by the same rat during the CS. The other evidence consists of forthcoming studies from our laboratory, in which both approach to the signal and to the food delivery site were measured during a CS for food by means of a segmented floor panel arrangement. These experiments show that under a paired CS-food arrangement similar to that used in the present study, rats displayed increases, at least initially, in both types of approach behavior. However, as in Phase 1, little or no persistent contact with the lighted response-key CS was obtained.

If, as our own data suggest, paired CS-food relations do in fact increase tendencies to approach the signal under circumstances like those of Phase 1, why is increased contact with the CS not observed also? Perhaps the answer lies in an explanation suggested by the outcome of the final test phase in this study. During this stage, paired rats were observed to contact (i.e., press) the CS key significantly more often than were unpaired rats. Since the subjects in both groups at this point had been exposed to no further differential treatment beyond that administered in Phase 1, this result may be interpreted as an indication that, even in the first conditioning stage, the potential for differential contact with the CS had been established. According to this view, the instrumental keypress training in Phase 2 may have provided the conditions necessary for the translation of this behavior potential into overt action. That is, tendencies to approach a signal for food may not eventuate in a specific topographical form of terminal contact with the stimulus unless the contact behavior itself is an innate or instrumentally conditioned component of the organism's normal sequence of food-getting behaviors in a particular situation. Perhaps the general success of autoshaping in the rat with protruding lever CSs that are paired with food is an indication of the importance of manipulative tendencies in the usual food-getting sequence of this species. Some support for this possibility can be inferred from the finding of Peterson et al. (1972) that a lever CS paired with brain stimulation rather than food also leads to approach but not much signal contact in the rat. In any case, while approach (or withdrawal) from stimuli correlated with reinforcement may be a ubiquitous consequence of exposure to stimulus-reinforcer relations, the form of terminal contact may be determined largely by species characteristics, signal properties, the nature of the reinforcer used (cf. Hearst \& Jenkins, 1974), and/or by prior exposure to response-reinforcer relations that have augmented the organism's normal behavioral repertoire (see Boakes, 1977, for a similar argument).

It must be acknowledged, however, that the latter interpretation of the present study is predicated upon the assumption that the observed differential choice behavior in Phase 3 can be attributed exclusively to disparate response evoking properties of the CS key. Clearly, such would be the case only if Phase 2 instrumental training was equally effective for both groups in establishing directed behavior to the $S^{D}$ key light. But an interaction between Phase 1 and Phase 2 treatments could result in $S^{D}$ properties that differed for paired and unpaired groups. Consequently, a pattern of results such as those obtained in Phase 3 could occur even when the response directing properties of the CS were equivalent in both groups. Although this possibility cannot be ruled out of the present study, it is not supported by the finding of apparently equivalent acquisition of FR 6 keypressing in Phase 2. Therefore, in the absence of more definitive evidence in this matter, the present conclusions must be regarded as tentative.

\section{REFERENCES}

Boakes, R. A. Performance on learning to associate a stimulus with positive reinforcement. In H. Davis \& H. M. B. Hurwitz (Eds.), Operant-Pavlovian interactions. Hillsdale, N.J: Erlbaum, 1977.

Brown, P. L., \& Jenkins, H. M. Auto-shaping of the pigeon's key-peck. Journal of the Experimental Analysis of Behavior, $1968,11,1-8$.

Crowell, C. R., \& Bernhardt, T. P. The feature-positive effect and sign-tracking behavior during discrimination learning in the rat. Animal Learning \& Behavior, 1979, 1, 313-317.

Hearst, E. Pavlovian conditioning and directed movements. In G. Bower (Ed.), The psychology of learning and motivation (Vol. 9). New York: Academic Press, 1975.

HEARST, E. Stimulus relationships and feature selection in learning and behavior. In S. H. Hulse, H. Fowler, \& W. K. Honig (Eds.), Cognitive processes in animal behavior. Hillsdale, N.J: Erlbaum, 1978.

Hearst, E., \& Jenkins, H. M. Sign-tracking: The stimulus reinforcer relation and directed action. Austin, Tex: Psychonomic Society, 1974.

Locurto, C., Terrace, H. S., \& Gibbon, J. Auto-shaping, random control, and omission training in the rat. Journal of the Experimental Analysis of Behavior, 1976, 26, 451-462.

Peterson, G. B., Ackil, J., Frommer, G., \& Hearst, E. Conditioned approach and contact behaviors toward signals for food or brain-stimulation reinforcement. Science, 1972, 177, 1009-1011.

Schwartz, B., \& Gamzu, E. Pavlovian control of operant behavior: An analysis of autoshaping and its implications for operant conditioning. In W. K. Honig \& J. E. R. Staddon (Eds.), Handbook of operant behavior. New York: Prentice-Hall, 1977.

(Received for publication December 15, 1980.) 